\title{
Flare-induced decay-less transverse oscillations in solar coronal loops ${ }^{\star}$
}

\author{
Sudip Mandal ${ }^{1} \oplus$, Hui Tian ${ }^{2,3}$, and Hardi Peter ${ }^{1}$ \\ 1 Max Planck Institute for Solar System Research, Justus-von-Liebig-Weg 3, 37077 Göttingen, Germany \\ e-mail: smandal.solar@gmail.com \\ 2 School of Earth and Space Sciences, Peking University, Beijing 100871, PR China \\ 3 Key Laboratory of Solar Activity, National Astronomical Observatories, Chinese Academy of Sciences, Beijing 100012, PR China
}

Received 14 June 2021 / Accepted 5 July 2021

\begin{abstract}
Evidence of flare-induced, large-amplitude, decay-less transverse oscillations is presented. A system of multithermal coronal loops, as observed with the Atmospheric Imaging Assembly (AIA), exhibit decay-less transverse oscillations after a flare erupts nearby one of the loop footpoints. Measured oscillation periods lie between $4.2 \mathrm{~min}$ and $6.9 \mathrm{~min}$ wherein the displacement amplitudes range from $0.17 \mathrm{Mm}$ to $1.16 \mathrm{Mm}$. A motion-magnification technique has been employed to detect the preflare decay-less oscillations. These oscillations have similar periods (between $3.7 \mathrm{~min}$ and $5.0 \mathrm{~min}$ ) similar to the previous ones, but their amplitudes $(0.04 \mathrm{Mm}$ to $0.12 \mathrm{Mm})$ are found to be significantly smaller. No phase difference is found among oscillating threads of a loop when observed through a particular AIA channel or when their multichannel signatures are compared. These features suggest that the occurrence of a flare in this case neither changed the nature of these oscillations (decaying versus decay-less), nor the oscillation periods. The only effect the flare has is to increase the oscillation amplitudes.
\end{abstract}

Key words. Sun: activity - Sun: corona - Sun: magnetic fields - Sun: oscillations - Sun: flares

\section{Introduction}

The solar corona, a highly structured million Kelvin hot and dynamic upper atmosphere of the Sun, accommodates a wide variety of magnetic structures including coronal loops. These loops act as wave guides for a range of magnetohydrodynamical (MHD) waves that are observed in the corona (Nakariakov \& Verwichte 2005). One such wave mode is the transverse or kink oscillation. Since the time of its discovery (Aschwanden et al. 1999; Nakariakov et al. 1999), kink waves have been routinely identified as transverse loop displacements in extreme ultraviolet (EUV) images (see Ruderman \& Erdélyi 2009; Nakariakov \& Kolotkov 2020 for detailed reviews). Over the course of various observations, it has been found that these kink waves are often triggered by a nearby transient event, such as a flare (Schrijver et al. 2002) or a jet (Zimovets \& Nakariakov 2015), or by a coronal mass ejection (CME; Wang et al. 2012). Furthermore, once triggered, these waves often die down rapidly (within a couple of wave periods). This rapid decay of the wave amplitude has now been established as a key observational feature of kink waves (Aschwanden et al. 2002). In MHD wave theory, these oscillations are described as the fast magnetoacoustic wave mode which is weakly compressible in the long wavelength limit (Edwin \& Roberts 1983; Roberts et al. 1984; Van Doorsselaere et al. 2008). Further, the observed rapid damping is explained through a mechanism known as resonant absorption (Goossens et al. 2002, 2011; Ofman \& Aschwanden 2002).

Over the last decade or so, the availability of high-resolution and high-cadence EUV data, such as from the Atmospheric

\footnotetext{
^ Movie is available at https://www . aanda.org
}

Imaging Assembly (AIA; Lemen et al. 2012), has significantly improved our understanding of kink oscillations. In fact, it has also led to the discovery of a new kind of transverse oscillations, termed as "decay-less" kink oscillations (Tian et al. 2012; Wang et al. 2012; Nisticò et al. 2013). These are small amplitude $(\leq 0.5 \mathrm{Mm})$, transverse oscillations with periods between 3 and $7 \mathrm{~min}$ and they show no apparent decay in their amplitudes over a large number of wave periods. These waves are rather omnipresent in active region coronal loops and seem to have no apparent connection with any transient events such as a flare ${ }^{1}$ (Anfinogentov et al. 2015). This is particularly different from the traditionally observed, rapidly decaying kink waves which are always associated with an impulsive driver. Recent theoretical studies as well as MHD simulations on decay-less kink waves have hypothesized that these oscillations are driven either by the random footpoint motions (Nisticò et al. 2013; Nakariakov et al. 2016; Afanasyev et al. 2020) or through a monoperiodic driver (Afanasyev et al. 2019). However, an exact match with the observed wave properties has not been achieved yet.

In this Letter, we present a unique event where several multithermal coronal loops exhibit decay-less transverse oscillations that are triggered by a nearby flare. In Sect. 2 we describe the event and the data, whereas Sect. 3 outlines the results. Finally, we conclude by summarizing our findings in Sect. 4.

\footnotetext{
1 Although almost all decay-less oscillations have no connection to eruptive events, Wang et al. (2012) reported an event triggered by a CME
} 

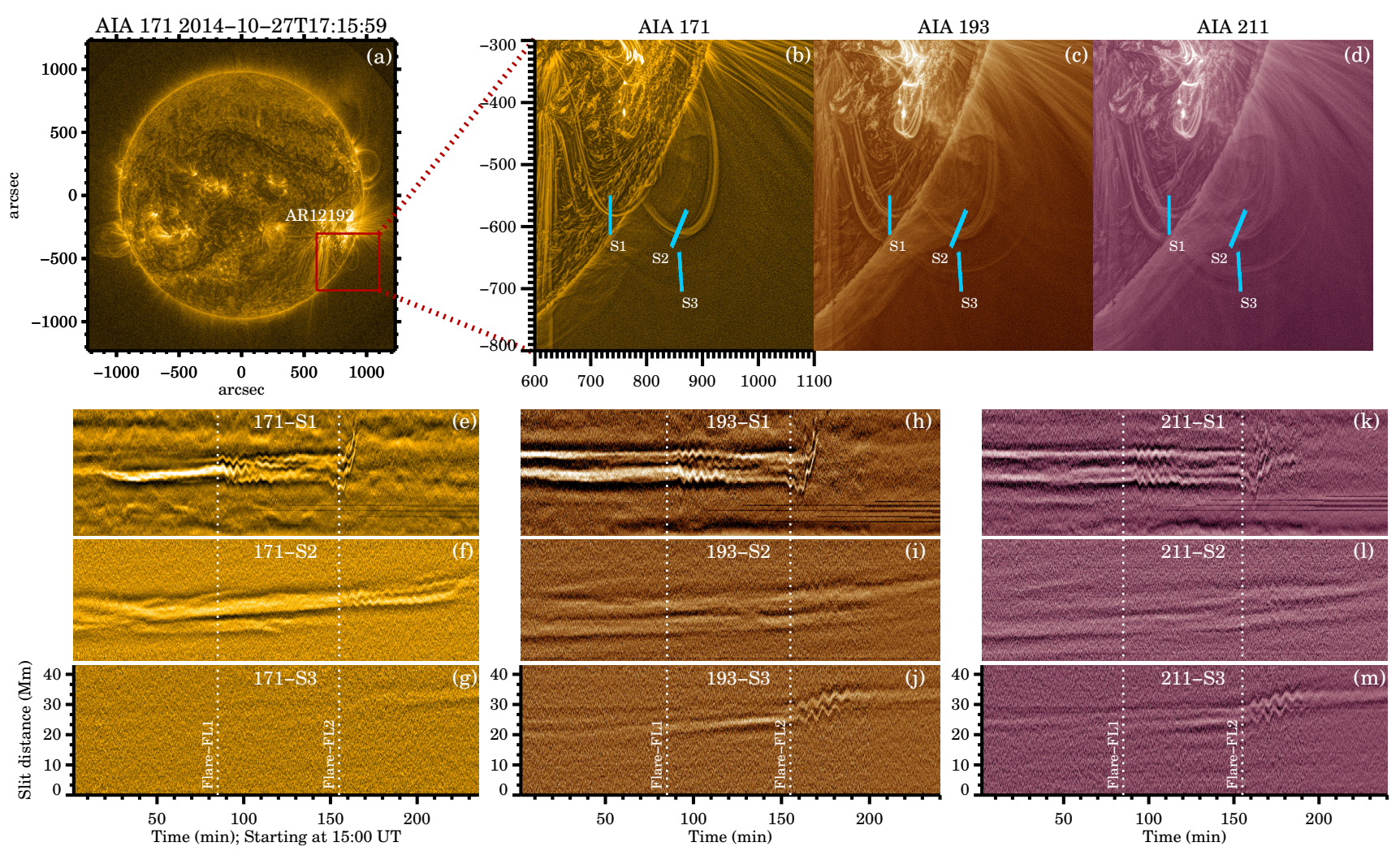

Fig. 1. Overview of oscillating loops and their temporal evolution. Panel a: a representative full-disk image of the Sun highlighting the location of our event by the red rectangular box. Images of this boxed region from $171 \AA, 193 \AA$, and $211 \AA$ channels are presented in panels $b-d$ respectively. Straight blue lines in each panel mark the locations of the three artificial slits (S1, S2, S3), which were used to generate the space-time (XT) maps. Generated XT maps are shown in panels $e-m$. Two vertical dotted lines in every panel mark the onset time of the two flares.

\section{Data and event details}

In this study, we use high-resolution extreme ultraviolet (EUV) imaging data from the AIA Lemen et al. (2012), onboard Solar Dynamics Observatory (SDO; Pesnell et al. 2012). Images from three different EUV passbands, namely the $171 \AA$, $193 \AA$, and $211 \AA$ channels, have been analyzed. The data we present here cover $240 \mathrm{~min}$ (starting from 15:00 UT on 27 October 2014) with a cadence of $12 \mathrm{~s}$. The pixel scale in each direction is $\approx 0.6^{\prime \prime}$.

The event occurred within a system of coronal loops that are rooted inside the active region AR 12192. This active region was located near the southwest limb of the Sun as shown in Fig. 1a. As is the case with any typical AIA EUV image, the off-limb coronal loops in our data appear to be fuzzy. This happens primarily due to the diffuse nature of the EUV emission. In order to accurately infer the nature of the observed oscillations, it is therefore important that we correctly identify the individual loops (and their strands). To bring out these types of finer details, we used the multi-Gaussian normalization (MGN) image processing technique as developed by Morgan \& Druckmüller (2014). As described in that paper, the MGN method basically normalizes an image at multiple spatial scales and also flattens the noisy regions, highlighting the small-scale details. All the data from $171 \AA, 193 \AA$, and $211 \AA$ channels are processed using this MGN method and the images presented in Figs. 1b-d are some of the examples of this processing. A quick look at these panels reveals two important aspects about the structure of these loops. Firstly, each loop is made up of multiple individual strands and, secondly, although most of these loops can visibly be traced in all three AIA channels, their contrasts are significantly different across these channels. In fact, some of these loops are even absent in the $171 \AA$ channel and only visible in the hotter $193 \AA$ or $211 \AA$ channel, implying that these structures are probably 1.5 MK to $2 \mathrm{MK}$ hot and thus, not prominent in the $171 \AA$ channel. All of these characteristics are basically a manifestation of the multithermal nature of the loop plasma.

\section{Results and discussion}

From the movie (available online) we can see that around 16:30 UT, a flare erupts close to the left footpoint of this loop system (see Sect. 2) and it subsequently triggers oscillations in the nearby loops. These oscillations continue to exist until about 17:40 UT, when we can see a second flare erupting at the exact same location as the previous one. This second flare is visibly more intense and as a result, it completely disrupts the nearby loops that were oscillating until then. However, some of the far away loops survived this flare outburst and they started oscillating thereafter.

\subsection{AIA light curves}

We analyzed the AIA light curves to better understand the flaring activities that we see near the loop footpoint. The blue box, drawn on top of the $171 \AA$ image (Fig. 2a), outlines our region of interest that includes the flaring site as well as the left loop footpoint. Light curves for each of the three AIA channels were then generated by averaging the intensity ${ }^{2}$ values over this box.

\footnotetext{
2 These intensities correspond to the original AIA measurements, i.e.,
} before the MGN processing. 


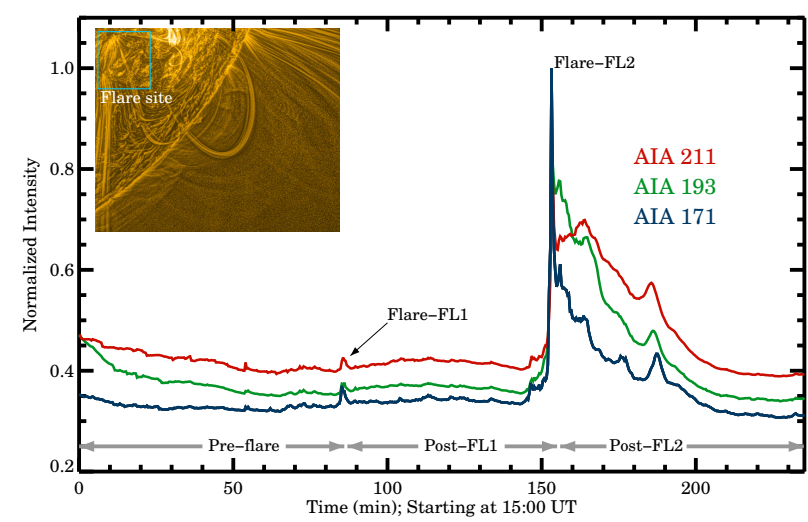

Fig. 2. Light curves from three different AIA channels. The inset shows the flaring site outlined by the blue box drawn on top of the $171 \AA$ image. Normalized intensity curves extracted from this boxed region are shown in the main panel. The two observed flares are also highlighted in the panel.

These curves were further normalized by their corresponding maximas and the final values are presented in Fig. 2b. From the plot, we find that there are no significant activities in any of these AIA channels during initial times. The first flare, FL1, erupts around $t \approx 85 \mathrm{~min}$ and can be seen as near simultaneous intensity enhancements in all three light curves. This flare lasts only about $3 \mathrm{~min}$ and another quiet period follows thereafter. Things, however, change rapidly around $t \approx 150 \mathrm{~min}$, marking the onset of the second flare, FL2. This flare is significantly stronger than FL1 (the AIA intensities in all three channels saturate during the peak of this flare). Additionally, there are multiple small intensity enhancements riding over these light curves during the decay phase of FL2. Overall, using these characteristics, we could divide our data into three activity phases, that is preflare phase, post-FL1 phase, and post-FL2 phase.

\subsection{Space-time maps}

To generate the space-time (XT) maps, we used multiple artificial transverse slits. The straight blue lines in Figs. 1b-d mark the locations of these slits (S1, S2, S3) wherein the corresponding XT maps are shown in Figs. 1e-m. In order to increase the intensity signal, we chose each of these slits to be 7 pixels wide for S1 and S2 and 9 pixels wide for S3. The final XT maps were then generated by averaging over their corresponding slit widths. Furthermore, for a chosen loop, we generated multiple XT maps by placing a series of slits and then we manually selected the best one where the oscillation signatures appear to be clearest by eye estimation.

\subsubsection{Postflare oscillations}

We first focused our attention to the oscillations that appear in the XT maps post-FL1 and FL2. In all of these maps (Figs. 1e-m), one can immediately notice the decay-less nature of most of these oscillations ${ }^{3}$, that is to say no apparent decay in their amplitudes even after several wave periods. In extreme cases (e.g., Figs. 1e,f,h,k), the amplitudes, in fact, do not show any decaying signature even after $50 \mathrm{~min}$ post-FL1. We now analyze each of these individual XT maps. In the case of the

\footnotetext{
3 We do note that the oscillation in the central thread in Fig. 1e appears more similar to a slowly decaying one.
}

maps for slit S1, oscillations appear immediately after FL1 $(t \approx 88 \mathrm{~min})$ and are prominently visible in all three AIA channels. On the other hand, oscillations in S2 only start after FL2 ( $t \approx 153 \mathrm{~min}$ ). This time the oscillatory signatures are best seen in the $171 \AA$ channel and they are faintly visible in the other two channels. The scenario, however, reverses in S3 maps where we find clear oscillations (which again appear only after FL2) in the hotter $193 \AA$ and $211 \AA$ channels and a significantly weaker signal in the $171 \AA$ channel.

Next, we proceeded to fitting these individual oscillations. For each oscillating strand, we first determined its "skeleton" by fitting a Gaussian along the transverse direction of these maps. The centers of these fitted Gaussians were then grouped together to constitute a skeleton. In each panel of Fig. 3, we highlight these centers by the red circles. A combination of a sine function and a linear trend

$y(t)=A *\left(\sin \frac{2 \pi t}{\mathrm{P}}+\phi\right)+c_{1} t+c_{0}$

was then fitted to these individual skeletons (belonging to a particular thread). Here $\mathrm{A}$ is the oscillation amplitude, $\mathrm{P}$ represents the period, $\phi$ is the phase, and $c_{0}$ and $c_{1}$ are constants.

The final fits to the detected oscillations are overplotted by the blue curves in different panels of Fig. 3. The fitted periods (P) and amplitudes (A) of all detected oscillations are listed in Table 1 (post-FL1 and post-FL2 columns). From the table, we note that the wave periods lie between $4.2 \mathrm{~min}$ and $6.9 \mathrm{~min}$, with the majority of them being close to $4.5 \mathrm{~min}$. This is consistent with the previous reports of decay-less oscillations (Anfinogentov et al. 2013, 2015). The amplitude values, on the other hand, show a broader distribution with values between $0.17 \mathrm{Mm}$ and $1.16 \mathrm{Mm}$. Interestingly, the majority of these A values are considerably larger than typical amplitude values we see in decay-less oscillations (Anfinogentov et al. 2015).

\subsubsection{Preflare oscillations}

Previous reports of decay-less kink waves highlighted the fact that these oscillations are omnipresent in coronal loops and have no apparent association with flares (Tian et al. 2012; Anfinogentov et al. 2015). Thus, it is natural for us to look for the signatures of such oscillations in the pre-flare phase data of our event. As shown earlier, the data before the first flare (i.e., before 16:29 UT) are considered to be the "preflare" data in this case.

A careful inspection of each of the original XT diagrams (Figs. 1e-m) reveals that out of all of these maps, definite signatures of such small amplitude, preflare oscillations are only visible in the XT maps of slit S1 of the $171 \AA$ and $193 \AA$ channels and they are rather faintly visible in the XT maps of slits S2 of the $171 \AA$ channel (see Figs. 1e,g,h). Although these oscillatory patterns can be traced visually, their signals are not sufficient to perform any reliable fit. Thus, we used a "motion magnification" technique (Anfinogentov \& Nakariakov 2016) to enhance these oscillation signals. This technique uses a dual-tree complex wavelet transform to amplify the signals of transverse motions that are present within an image sequence. The right panels of Fig. 4 show the original (non-magnified) XT maps, wherein the preflare motion magnified XT maps are shown in the left panels. We tested different magnification factors $(K)$ between 3 and 10, and after visual inspection, we settled on a value of $K=5$ to best enhance the oscillation signal. 


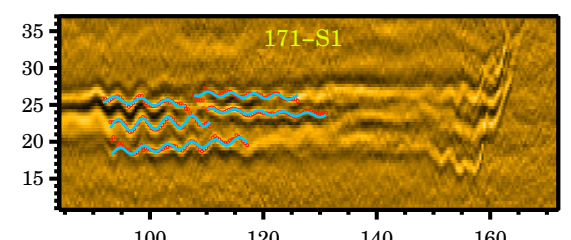

100

160
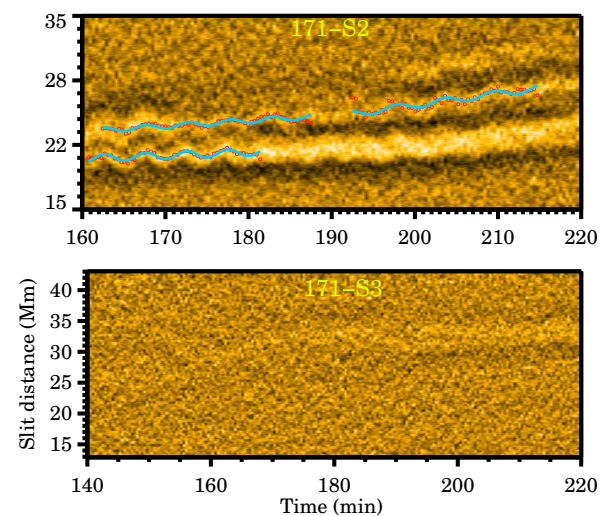
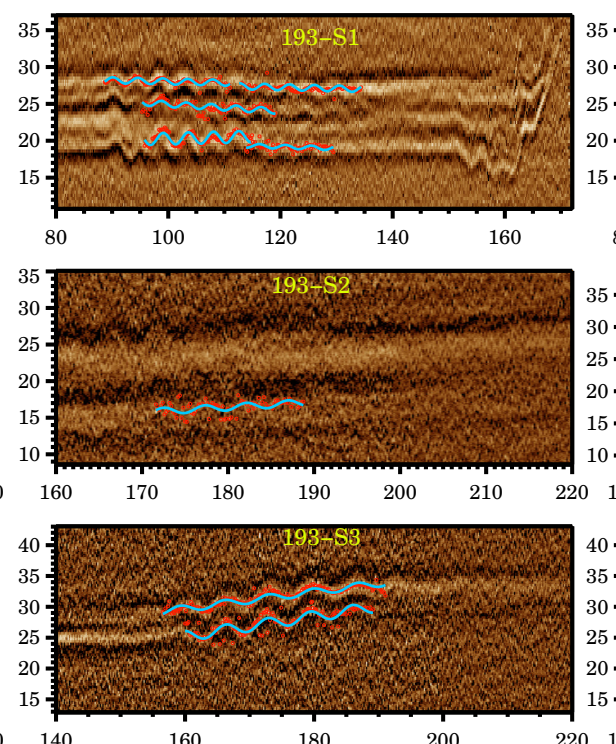
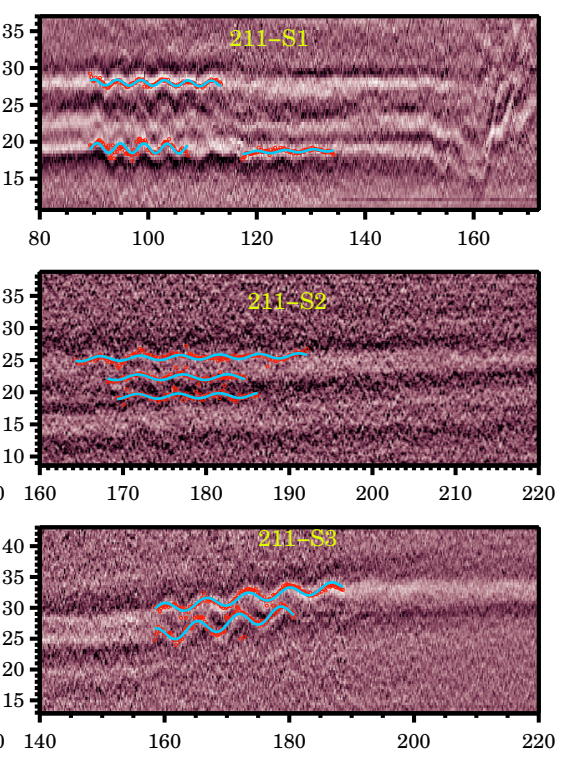

Fig. 3. Fits to the individual oscillations. The red points highlight the skeletons of the individual oscillating structures, whereas the best fitting sinusoidal function (Eq. (1)) is shown by the blue curves. See Sect. 3.2.1 for more details.

With these magnified XT maps, we can now better identify these preflare oscillations. Although the $193 \AA$ oscillations are somewhat blurry, the $171 \AA$ ones are prominent and their decayless nature is also very evident. An interesting feature to note here is the shape of these oscillatory signals, which appears to be pointier or more triangle-shaped than sinusoidal. Similar characteristics were also seen in the examples presented in Anfinogentov et al. (2013), Antolin et al. (2016). We measured the wave periods and amplitudes in the same way as discussed earlier and the best fits to the observed oscillations are shown by the blue curves in Fig. 4. Derived period and amplitude values are listed in the preflare columns of Table 1. Looking at this table, we notice that the preflare periods are similar to those of the postflare values. At the same time, the preflare amplitudes are significantly smaller (approximately 3 to 5 times) than the postflare ones. Interestingly, our preflare amplitudes match well with the amplitude values reported in previous studies of small amplitude decay-less oscillations (Anfinogentov et al. 2015). More discussion on this is presented in the next section.

\subsubsection{Comparison between the preflare and postflare oscillations}

In the discussion of our study, so far we have described that these decay-less oscillations continue to exist in a loop despite multiple flaring events occurring nearby. In order to better understand the impact of these flares on the observed oscillations, we compare the preflare and postflare wave properties here. Figure 5 shows the period-amplitude diagram that was constructed using all the individual oscillations that are listed in Table 1. In this plot, each data point is marked with a color that indicates its slit of origin, whereas the associated symbol represents its epoch of occurrence. From the period histogram (top panel of Fig. 5), we notice that the oscillation periods (both pre- and postflare ones) are mostly restricted between 4 and $6 \mathrm{~min}$, although the S3 periods are grouped near $P=7 \mathrm{~min}$. These longer periods occur only in the space-time plots of S3. This is clear because this particular slit, S3, was placed onto the large distant loop, and the kink period $(\mathrm{P})$ increases linearly with loop lengths
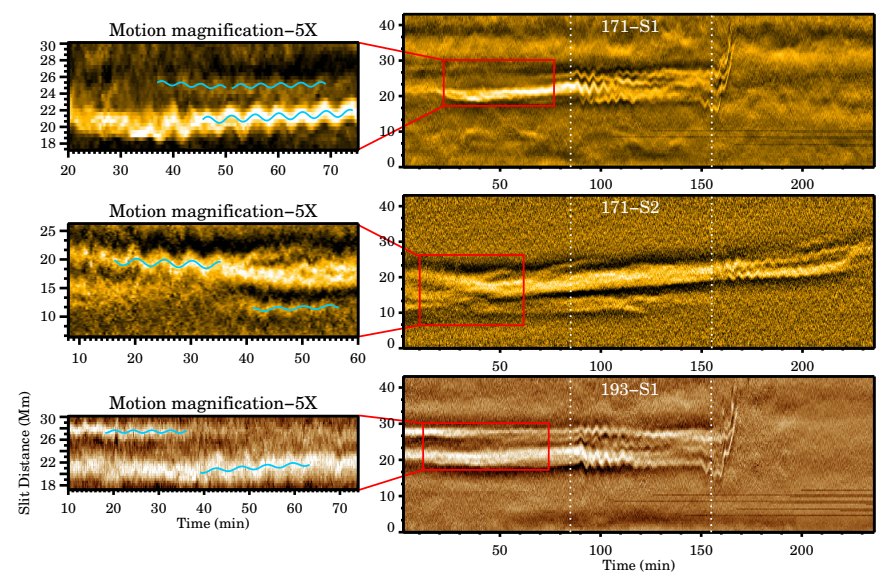

Fig. 4. Preflare oscillation maps. Right panels (top to bottom): spacetime (XT) maps from slits 171-S1, 171-S2, and 193-S1. In each case, a red rectangular box outlines the preflare phase of the data that is used to generate the corresponding motion magnified map. Left panels: motion magnified maps for the abovementioned three slits. The blue curves show the best fitting sinusoidal function to the detected oscillatory patterns. See Sect. 3.2.2.

(Anfinogentov et al. 2015). The distribution of wave amplitudes (A) show some interesting features. All the preflare values are either below or very close to $0.1 \mathrm{Mm}$, whereas post-FL1 A values lie between $0.15 \mathrm{Mm}$ and $0.8 \mathrm{Mm}$, with the majority of them clustering around $0.4 \mathrm{Mm}$. Thus, on average, the post-FL1 oscillations have 5 times larger amplitudes than the preflare ones, although the wave periods mostly remained unchanged between these two epochs. Looking at this trend, one would be inclined to conclude that this enhancement in wave amplitudes is directly proportional to the strength of the triggering flare. However, when we included the post-FL2 oscillations, such a relation did not hold strong. For example, the post-FL2 S2 amplitudes are similar to that of the post-FL1 S1 oscillations, whereas the postFL2 S3 amplitudes are bigger. Hence, oscillations of different amplitudes are generated by a single flaring event. In the same 
Table 1. Fitted parameters of individual wave threads.

\begin{tabular}{|c|c|c|c|c|c|c|c|}
\hline \multirow[b]{2}{*}{ Slit } & \multirow[b]{2}{*}{ Channel } & \multicolumn{2}{|c|}{ Preflare } & \multicolumn{2}{|c|}{ Post-FL1 } & \multicolumn{2}{|c|}{ Post-FL2 } \\
\hline & & $\mathrm{P}(\mathrm{min})$ & $\mathrm{A}(\mathrm{Mm})$ & $\mathrm{P}(\min )$ & $\mathrm{A}(\mathrm{Mm})$ & $\mathrm{P}(\min )$ & $\mathrm{A}(\mathrm{Mm})$ \\
\hline \multirow{5}{*}{$\mathrm{S} 1$} & \multirow{5}{*}{171} & 3.7 & 0.04 & 4.2 & 0.33 & - & - \\
\hline & & 4.5 & 0.08 & 4.5 & 0.28 & - & - \\
\hline & & 3.8 & 0.06 & 4.6 & 0.80 & - & - \\
\hline & & - & - & 4.4 & 0.50 & - & - \\
\hline & & - & - & 4.8 & 0.45 & - & - \\
\hline \multirow{6}{*}{$\mathrm{S} 1$} & \multirow{6}{*}{193} & 4.4 & 0.05 & 4.4 & 0.41 & - & - \\
\hline & & 5.6 & 0.07 & 4.4 & 0.38 & - & - \\
\hline & & - & - & 4.5 & 0.80 & - & - \\
\hline & & - & - & 4.5 & 0.17 & - & - \\
\hline & & - & - & 4.6 & 0.27 & - & - \\
\hline & & - & - & 5.8 & 0.36 & - & - \\
\hline \multirow{3}{*}{$\mathrm{S} 1$} & \multirow{3}{*}{211} & - & - & 4.3 & 0.39 & - & - \\
\hline & & - & - & 4.3 & 0.66 & - & - \\
\hline & & - & - & 5.2 & 0.18 & - & - \\
\hline \multirow{3}{*}{ S2 } & \multirow{3}{*}{171} & 4.6 & 0.06 & - & - & 5.0 & 0.38 \\
\hline & & 5.0 & 0.12 & - & - & 5.1 & 0.26 \\
\hline & & - & - & - & - & 5.9 & 0.33 \\
\hline \multirow[t]{2}{*}{$\underline{\mathrm{S} 2}$} & 193 & - & - & - & - & 4.8 & 0.45 \\
\hline & & - & - & - & - & 4.8 & 0.37 \\
\hline \multirow[t]{2}{*}{$\mathrm{S} 2$} & 211 & - & - & - & - & 5.4 & 0.44 \\
\hline & & - & - & - & - & 4.9 & 0.39 \\
\hline S3 & 171 & - & - & - & - & - & - \\
\hline \multirow[t]{2}{*}{$\overline{\mathrm{S} 3}$} & 193 & - & - & - & - & 6.9 & 0.55 \\
\hline & & - & - & - & - & 6.6 & 0.87 \\
\hline$\overline{\mathrm{S} 3}$ & 211 & - & - & - & - & 6.7 & 0.83 \\
\hline- & - & - & - & - & - & 6.7 & 1.16 \\
\hline
\end{tabular}

Notes. Typical fitting error in $\mathrm{P}$ is $\pm 0.1 \mathrm{~min}$, whereas the same in $\mathrm{A}$ is $\pm 0.02 \mathrm{Mm}$.

context, we again recall that the flare FL2, which triggered these oscillations, is a significantly stronger flare than FL1. From this we can conclude that the occurrence of a flare in this case neither changed the nature of these oscillations nor the oscillation periods, but it increased their amplitudes.

\subsubsection{Multiwavelength aspect}

In this section, we examine the multiwavelength properties of these oscillations. To do this, we selected the time-distance maps from slit $\mathrm{S} 1$ as the oscillation signals in these maps, which are prominently visible in all three AIA channels. Through different panels of Fig. 6, we show the post-FL1 period of the S1 maps from $171 \AA, 193 \AA$, and $211 \AA$. Further, the individual threads (Ts) are also marked with dotted horizontal lines. By following these threads, we find that these oscillatory patterns start at exactly the same time in all three AIA channels. Additionally, we also notice that although these oscillations appear sharper (i.e., with better contrasts) in the beginning, they become rather diffuse at later times. This is possibly an artifact of the movements of these individual threads. Moreover, a closer inspection also reveals that the thread T1, which is visible throughout the $193 \AA$ and $211 \AA$ channels, becomes visible in the $171 \AA$ channel only after $t=110 \mathrm{~min}$. On the other hand, the thread T2 only appears in channels $171 \AA$ and $193 \AA$ and is absent in the $211 \AA$ channel. These are basically the manifestations of the thermal structuring of the loop plasma.

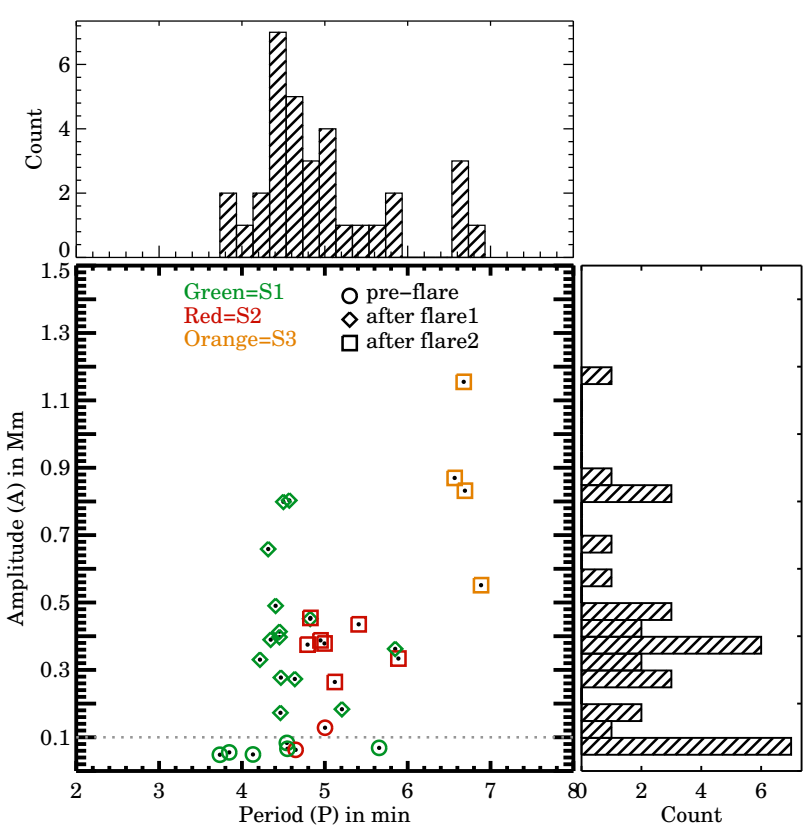

Fig. 5. Period-amplitude diagram. Colors (green, red, orange) indicate the slits $(\mathrm{S} 1, \mathrm{~S} 2, \mathrm{~S} 3)$ from which these oscillations originate, whereas the symbols (circle, diamond, square) illustrate the epoch (preflare, post-FL1, post-FL2) of these oscillations. The dotted horizontal line in the plot marks the $A=0.1 \mathrm{Mm}$ boundary. Top and side panels: histograms of period $(\mathrm{P})$ and amplitude $(\mathrm{A})$ values.

Some of the earlier studies (e.g., Wang et al. 2012; Antolin et al. 2016) had found a phase difference between such oscillations when observed simultaneously through two different AIA channels. Hence, we also looked for such signatures in our data. In Fig. 6, we mark the positions of the oscillation maximas at different wave cycles. From the plot, we see that the oscillation maximas appear simultaneously across all AIA channels. Further, this in-phase behavior persists even after multiple wave periods. Hence, we conclude that these oscillating threads, across multiple AIA channels, are moving in phase. Additionally, we found that every thread within a given space-time map also exhibits synchronous oscillation with all other threads in that map and again, this behavior remains unchanged during the entire lifetime of these oscillations. The in-phase oscillations seen in different AIA channels are consistent with the finding by Tian et al. (2012), where these authors found in-phase oscillations in different spectral lines observed with Hinode/EIS ${ }^{4}$.

\section{Summary and conclusion}

In this Letter, we study the properties of decay-less transverse oscillations that are triggered by a nearby flaring event. These oscillations appear within a system of coronal loops that are rooted inside an active region.

1. The observed oscillations are transverse in nature and they last for three to ten wave periods without any measurable decay in their amplitudes. These are basically the signatures of decay-less kink waves. The wave periods range from between $4.2 \mathrm{~min}$ and $6.9 \mathrm{~min}$, whereas the wave amplitudes lie within $0.17 \mathrm{Mm}$ and $1.16 \mathrm{Mm}$.

2. The observed decay-less oscillations are generated by a nearby flare, FL1. Once triggered, these waves continue to

4 Extreme-ultraviolet Imaging Spectrometer. 


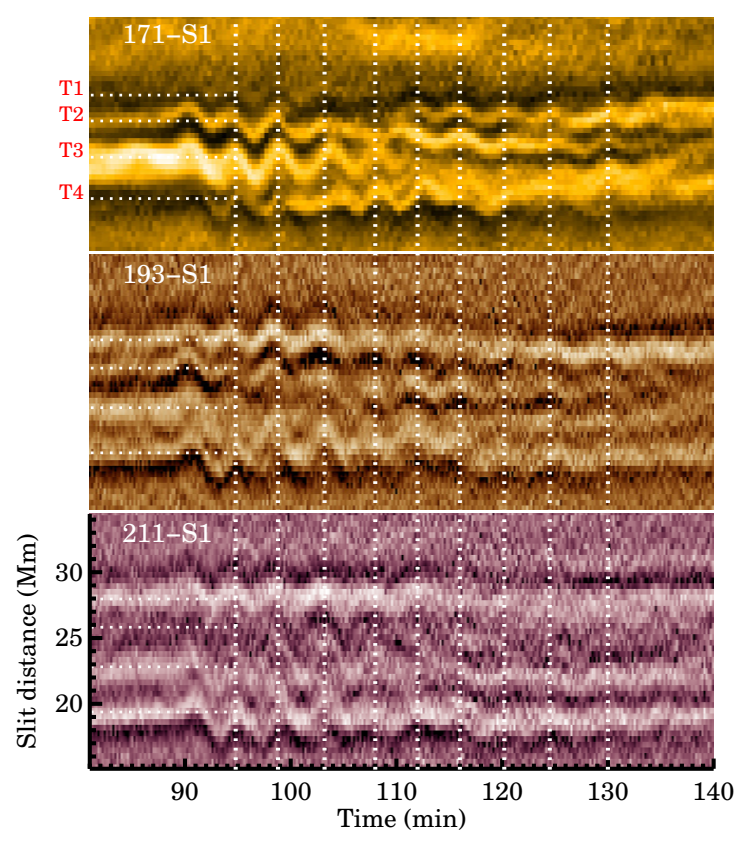

Fig. 6. Multiwavelength view of the $S 1$ oscillations. Vertical lines mark the positions of the wave maximas as seen in the $193 \AA$ channel. The horizontal lines highlight the initial locations of the individual loopthreads (T).

exist until another nearby flare, FL2, disrupts the oscillating loops. Multiwavelength analysis reveals a synchronous appearance of these oscillatory patterns in different AIA channels (171 $\AA$, $193 \AA, 211 \AA)$. In any given AIA channel, all loop threads oscillate in phase. A similar in-phase oscillation is also seen across different AIA channels.

3. Small amplitude, preflare, decay-less transverse oscillations are also present in our data. These waves exist within the same loop structures in which we previously noted the postflare oscillations. After classifying each oscillation according to it appearance in the data (i.e., preflare or postflare), a pattern emerges.

Our main finding is that the postflare oscillations are found to have 5 to 7 times bigger amplitudes than the preflare ones, whereas the oscillation periods, before and after the flare, remained similar.

The mechanism that drives these decay-less transverse oscillations is still not fully understood. In case of small amplitude preflare cases, some authors suggested random footpoint driving (caused by supergranular motions) to be the source (e.g., Nisticò et al. 2013; Nakariakov et al. 2016). The wide range of observed wave periods, however, does not match the driver characteristics. In a different proposal, Antolin et al. (2016) argued that the Kelvin-Helmholtz (KH) instabilities near the loop boundaries can potentially mimic the observed decay-less features. Interestingly, the postflare oscillations that we have found in the case of S2 of the $171 \AA$ channel (see Fig. 1f) look very similar to the synthesized XT maps that were presented in Fig. 3 of Antolin et al. (2016). However, the in-phase oscillations across three AIA channels, as seen in our data, would not be consistent with this mechanism. Hence, it is possible that the oscillations that we see in this study are probably governed by a combination of all of these individual mechanisms, that is, an impulsive driver (the flares), a perpetual driver (the footpoint motions), and the KH rolls. With the current spatial (and temporal) resolution of modern EUV imaging data, it is rather difficult to identify (and quantify) the individual contributions of each of these mechanisms. Nevertheless, Antolin et al. (2016) have recently demonstrated how a change in spatial resolution can affect the appearance of these oscillations in EUV images and in this context, future observations from the Extreme Ultraviolet Imager (EUI), on board Solar Orbiter, will be extremely useful in de-entangling certain aspects of these oscillations.

Acknowledgements. We thank the reviewer for his/her encouraging comments. S.M. acknowledges Prof. Dipankar Banerjee for his help in understanding the data. H.T. is supported by the Strategic Priority Research Program of CAS (grant XDA17040507) and NSFC grant 11825301. The AIA data used here is courtesy of the SDO (NASA) and AIA consortium. The authors would also like to acknowledge the Joint Science Operations Center (JSOC) for providing the AIA data download links.

\section{References}

Afanasyev, A., Karampelas, K., \& Van Doorsselaere, T. 2019, ApJ, 876, 100 Afanasyev, A. N., Van Doorsselaere, T., \& Nakariakov, V. M. 2020, A\&A, 633, L8

Anfinogentov, S., \& Nakariakov, V. M. 2016, Sol. Phys., 291, 3251

Anfinogentov, S., Nisticò, G., \& Nakariakov, V. M. 2013, A\&A, 560, A107

Anfinogentov, S. A., Nakariakov, V. M., \& Nisticò, G. 2015, A\&A, 583, A136

Antolin, P., De Moortel, I., Van Doorsselaere, T., \& Yokoyama, T. 2016, ApJ, 830, L22

Aschwanden, M. J., Fletcher, L., Schrijver, C. J., \& Alexander, D. 1999, ApJ, 520,880

Aschwanden, M. J., de Pontieu, B., Schrijver, C. J., \& Title, A. M. 2002, Sol. Phys., 206, 99

Edwin, P. M., \& Roberts, B. 1983, Sol. Phys., 88, 179

Goossens, M., Andries, J., \& Aschwanden, M. J. 2002, A\&A, 394, L39

Goossens, M., Erdélyi, R., \& Ruderman, M. S. 2011, Space Sci. Rev., 158, 289

Lemen, J. R., Title, A. M., Akin, D. J., et al. 2012, Sol. Phys., 275, 17

Morgan, H., \& Druckmüller, M. 2014, Sol. Phys., 289, 2945

Nakariakov, V. M., \& Kolotkov, D. Y. 2020, ARA\&A, 58, 441

Nakariakov, V. M., \& Verwichte, E. 2005, Liv. Rev. Sol. Phys., 2, 3

Nakariakov, V. M., Ofman, L., Deluca, E. E., Roberts, B., \& Davila, J. M. 1999, Science, 285, 862

Nakariakov, V. M., Anfinogentov, S. A., Nisticò, G., \& Lee, D. H. 2016, A\&A, 591, L5

Nisticò, G., Nakariakov, V. M., \& Verwichte, E. 2013, A\&A, 552, A57

Ofman, L., \& Aschwanden, M. J. 2002, ApJ, 576, L153

Pesnell, W. D., Thompson, B. J., \& Chamberlin, P. C. 2012, Sol. Phys., 275, 3

Roberts, B., Edwin, P. M., \& Benz, A. O. 1984, ApJ, 279, 857

Ruderman, M. S., \& Erdélyi, R. 2009, Space Sci. Rev., 149, 199

Schrijver, C. J., Aschwanden, M. J., \& Title, A. M. 2002, Sol. Phys., 206, 69

Tian, H., McIntosh, S. W., Wang, T., et al. 2012, ApJ, 759, 144

Van Doorsselaere, T., Nakariakov, V. M., \& Verwichte, E. 2008, ApJ, 676, L73

Wang, T., Ofman, L., Davila, J. M., \& Su, Y. 2012, ApJ, 751, L27

Zimovets, I. V., \& Nakariakov, V. M. 2015, A\&A, 577, A4 